\title{
The CALorimetric Electron Telescope (CALET) on the ISS: Preliminary Results from On-orbit Observations since October, 2015
}

\author{
Shoji Torii for the CALET Collaboration* \\ Research Institute for Science and Engineering , Waseda Waseda University, 3-4-1 Okubo, \\ Shinjuku, Tokyo 169-8555, Japan \\ E-mail: torii.shojiewaseda.jp
}

The CALorimetric Electron Telescope (CALET) space experiment, which has been developed by Japan in collaboration with Italy and the United States, is a high-energy astroparticle physics mission on the International Space Station (ISS). The primary goals of the CALET mission include investigating the presence of possible nearby sources of high-energy electrons, studying the details of galactic particle propagation and searching for dark-matter signatures. During a two-year mission, extendable to five years, the CALET experiment is measuring the flux of cosmic-ray electrons (including positrons) to $20 \mathrm{TeV}$, gamma-rays to $10 \mathrm{TeV}$ and nuclei with $\mathrm{Z}=1$ to 40 up to $1,000 \mathrm{TeV}$. In addition, gamma-ray burst observations in the $7 \mathrm{keV}$ to $20 \mathrm{MeV}$ range, including searches for the electromagnetic counterparts to LIGO-VIRGO gravitational events, are carried out. Solar modulation study by detection of the electrons below cut-off rigidity is also a target of the observations.

The instrument consists of two layers of segmented plastic scintillators for the cosmic-ray charge identification (CHD), a 3 radiation length thick tungsten/scintillating-fiber imaging calorimeter (IMC) and a 27 radiation length thick lead-tungstate calorimeter (TASC). CALET has sufficient depth, imaging capabilities and excellent energy resolution to allow for a clear separation between hadrons and electrons and between charged particles and gamma rays. The instrument was launched on August 19, 2015 to the ISS with HTV-5 (H2 Transfer Vehicle 5) and installed on the Japanese Experiment Module - Exposed Facility (JEM-EF) on August 25.

Since the start of operation in mid-October, 2015, continuous observation has been carried out without any major interruption, mainly by triggering on high-energy ( $>10 \mathrm{GeV})$ showers. The number of triggered events is about 20 million per month. By using the data obtained so far, we will present a summary of preliminary results from the CALET observations on 1) Electron energy spectrum, 2) Proton and Nuclei spectra, 3) Gamma-ray observations, with results of the on-orbit performance study.

35th International Cosmic Ray Conference

10-20 July, 2017

Bexco, Busan, Korea

* Speaker. 


\section{Introduction}

The CALorimetric Electron Telescope (CALET) is a Japan-led international mission funded by the Japanese Space Agency (JAXA) in collaboration with the Italian Space Agency (ASI) and NASA. The instrument was launched on August 19, 2015 by a Japanese carrier, H2 Transfer Vehicle (HTV), and robotically installed on the Japanese Experiment Module-Exposed Facility (JEM$\mathrm{EF}$ ) on the International Space Station (ISS) for a two-year mission, extendable to five years. Figure $\square$ shows CALET at the attach point \#9 on the JEM-EF. A schematic overview of the CALET instrument is presented in Fig. $\square$. The total weight is $613 \mathrm{~kg}$.
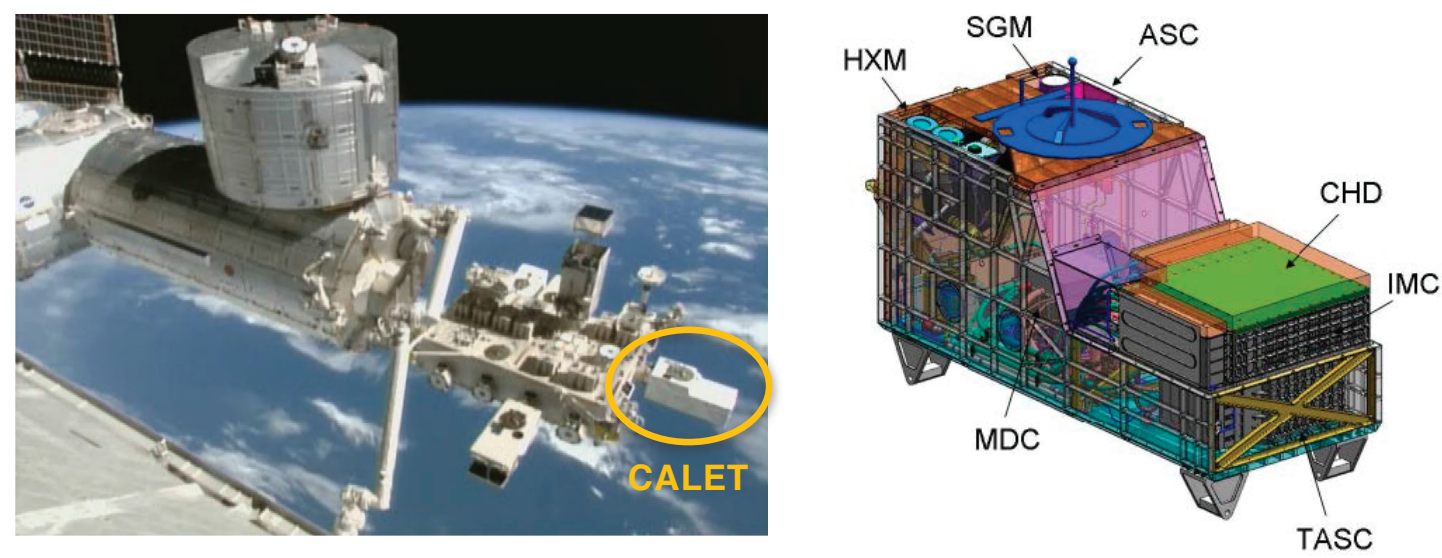

Figure 1: Japanese Experiment Module-Exposed Facility and CALET attached at the \#9 port .

Figure 2: CALET instrument package showing the main calorimeter composed of CHD, IMC and TASC (see $\S 2$ for the details), and CGBM subsystems [[13].

The primary science goal of CALET is to perform high-precision measurements of the cosmicray total electron spectrum from $1 \mathrm{GeV}$ to $20 \mathrm{TeV}$. In the high energy, $\mathrm{TeV}$, region, CALET can observe possible signatures of sources of high energy particle acceleration in our local region of the galaxy [四, [2]. In addition, the observed increase of the positron fraction over $10 \mathrm{GeV}$ by PAMELA [3] and AMS-02 [ [ [ 9 ] tells us that at high energy an unknown primary component of positrons may be present in addition to the secondary component produced during the galactic propagation process. Candidates for such primary sources range from astrophysical ones (e.g. Pulsar) to exotic (e.g. Dark Matter). Since these primary sources naturally emit positron-electron pairs, it is expected that the electron + positron (hereafter, all-electron) spectrum might exhibit a spectral structure determined by the origin of positrons. This may become visible in the high energy domain of the spectrum in the case, for instance, of an acceleration limit from pulsars or the mass of dark matter particles.

\section{Instrument}

CALET is an all-calorimetric instrument, with a total thickness equivalent to 30 radiation lengths $\left(\mathrm{X}_{0}\right)$ and 1.3 proton interaction lengths $\left(\lambda_{I}\right)$, preceded by a particle identification system. The unique feature of CALET is its thick, fully active calorimeter that allows measurements well into the TeV energy region with an excellent separation among hadrons and electrons $\left(\sim 10^{5}\right)$ and excellent energy resolution $(\sim 2 \%)$. The energy measurement relies on two kinds of calorimeters: a 
fine granulated pre-shower IMaging Calorimeter (IMC), followed by a Total AbSorption Calorimeter (TASC). In order to identify the individual chemical elements in the cosmic-ray flux, a Charge Detector (CHD) is placed at the top of the instrument.

Figure 31 presents a schematic side view of the calorimeter with a simulated shower profile produced by a $1 \mathrm{TeV}$ electron. An example of $1 \mathrm{TeV}$ electron candidate in the flight data is shown in Fig. $\mathbb{t}$ to give a real image in the detector space. CALET has a field of view of 45 degrees from the zenith, and the effective geometrical factor for high-energy electrons $(>10 \mathrm{GeV})$ is $\simeq 1040$ $\mathrm{cm}^{2} \mathrm{sr}$.

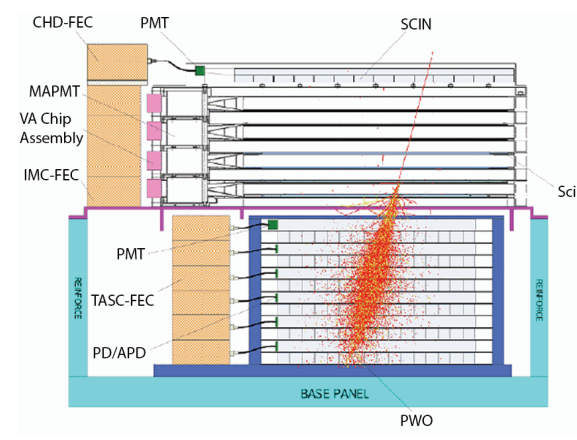

Figure 3: A schematic side view of the main calorimeter. An example of a simulation event for a $1 \mathrm{TeV}$ electron is superimposed to illustrate the shower development in the calorimeter.
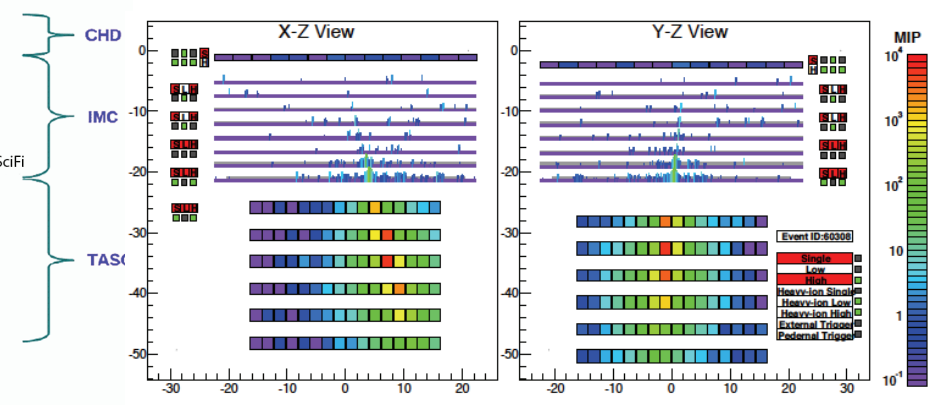

Figure 4: An example of observed $1 \mathrm{TeV}$ electron candidate presented on real-time event display. The scale presents the size of dimension in $\mathrm{cm}$. The colors give the signal value of each sensor in MIP, denoted in the right side.

The CHD has been designed to measure the charge of incoming charge via the $Z^{2}$ dependence of the specific ionization loss in a double layered, segmented, plastic scintillator array placed above the IMC. Each layer consists of 14 plastic scintillator paddles, with dimensions $450 \mathrm{~mm}(\mathrm{~L}) \times 32$ $\mathrm{mm}(\mathrm{W}) \times 10 \mathrm{~mm}(\mathrm{H})$. This segmented configuration has been optimized to reduce multi-hits on each paddle caused by backscattering particles. The two layers of paddles are orthogonally arranged to determine the incident position of cosmic-rays. The generated scintillation light is collected and read out by a photomultiplier tube (PMT). The CHD and related front-end electronics have been designed to provide incident particle identification over a large dynamic range for charges with $Z$ $=1 \sim 40$. Charge identification capabilities of the CHD have been measured by exposing it on an ion beam at GSI facility [5] and CERN-SPS [ [6], giving a charge resolution ranging from 0.15 electron charge units (e) for B to $0.30-0.35$ e in the Fe region.

The IMC images the early shower profile with a fine granularity by using $1 \mathrm{~mm}$ square cross section scintillating fibers (SciFi) individually read out by Multi-Anode Photomultiplier Tubes (64anode Hamamatsu R7600-M64). The imaging pre-shower consists of 7 layers of tungsten plates each separated by 2 layers of SciFi belts arranged in the $\mathrm{X}$ and $\mathrm{Y}$ direction and capped by an additional $X, Y$ SciFi layer pair. Each SciFi belt is assembled with 448 fibers with the dimensions of $448 \mathrm{~mm}(\mathrm{~L}) \times 448 \mathrm{~mm}(\mathrm{~W})$. The total thickness of the IMC is equivalent to $3 \mathrm{X}_{0}$. The first 5 tungsten-SciFi layers sample the shower every $0.2 \mathrm{X}_{0}$ and the last 2 layers provide $1.0 \mathrm{X}_{0}$ sampling. The IMC fine granularity allows to : (i) reconstruct the incident particle trajectory; (ii) determine the starting point of the shower; (iii) separate the incident particles from backscattered particles. 
Above $10 \mathrm{GeV}$, the expected angular resolution for gamma-rays is better than $\sim 0.4^{\circ}$, while the angular resolution for electrons is less than that of gamma-rays.

The homogeneous calorimeter is designed to measure the total energy of the incident particle and discriminate the electromagnetic from hadronic showers. TASC is composed of 12 layers, each consisting of 16 lead tungstate (PWO) logs. Each log has dimensions of $326 \mathrm{~mm}(\mathrm{~L}) \times 19$ $\mathrm{mm}(\mathrm{W}) \times 20 \mathrm{~mm}(\mathrm{H})$. Layers are alternately arranged with the logs oriented along orthogonal directions to provide a 3D reconstruction of the showers. Six layers image the XZ view and the other six the YZ view. The total area of the TASC is $326 \times 326 \mathrm{~mm}^{2}$ and the total thickness corresponds to about $27 \mathrm{X}_{0}$ and $1.2 \lambda_{I}$ in normal incidence. Each PWO log at the top layer is readout by the PMT to generate a trigger signal. Hybrid packages of silicon Avalanche Photo Diode and silicon Photo Diode (Dual APD/PD) are used to detect photons from all of the remaining PWO bars in the eleven layers. The readout front end system of each pair of the APD/PD sensors is configured with Charge Sensitive Amplifier (CSA) and pulse shaping amplifier with dual gain. Such a readout system provides a dynamic range covering 6 orders of magnitudes and make it possible to measure in each bar a signal spanning from 0.5 MIPs (Minimum Ionizing Particles) to $10^{6}$ MIPs, which is the energy deposit expected from a proton-induced $1000 \mathrm{TeV}$ shower.

The main scientific objective of CALET is the measurement of the electron spectrum over the range from $1 \mathrm{GeV}$ to $20 \mathrm{TeV}$. For this purpose, TASC is required to have a linear energy response from $\mathrm{GeV}$ up to the $\mathrm{TeV}$ region and an excellent resolution to resolve possible spectral features as expected in case of the presence of nearby CR sources or dark matter. According to Monte Carlo simulations and CERN-SPS beam test data, TASC can measure the energy of the incident electrons and gamma rays with resolution $\leq 2 \%$ above $100 \mathrm{GeV}$ [ [D]. Another necessary requirement is to efficiently identify high-energy electrons among the overwhelming background of CR protons. Particle identification information from both IMC and TASC is used to achieve an electron detection efficiency above $80 \%$ and a proton rejection power $\sim 10^{5}$ [Q, Q

In the region above $10 \mathrm{GeV}$, electrons and gamma rays are separated by the IMC, as gamma rays have no tracks in the IMC, except for backscattered particles. Furthermore, the charge measurements of the CHD can be used to reject charged particles. At energies less than $1 \mathrm{TeV}$, a gamma-ray rejection power (for electron observations) is expected to be $\sim 500$, while the electron rejection power (for gamma-ray observations) is better than $10^{4}$ [ए]].

\section{On-orbit operations}

The observation data obtained with CALET onboard ISS is transferred to JAXA via the NASA data-downlink system using the Tracking and Data Relay Satellite System (TDRSS). To operate and to monitor CALET, the JAXA Ground Support Equipment (JAXA-GSE) at the JAXA Tsukuba Space Center (TKSC) and the Waseda CALET Operations Center (WCOC) in Waseda University have been established. The real-time data received by JAXA-GSE are immediately transferred to WCOC. Scientific raw data are also transferred to WCOC on an hourly basis after time-order correction and completion of the replay data at JAXA-GSE. For the CALET project which aims at unique scientific goals by challenging the boundary of statistics and accuracy, it is very important to maintain detector performance and to carry out the observation with high efficiency during the long term operations. 
Planning the trigger conditions for various regions of the orbit takes account of geomagnetic latitude with the goal of efficient observation of low-energy particles and calibration data while achieving the highest possible statistics for $\mathrm{TeV}$ electrons. The following trigger modes are combined in on-orbit operations [ए]].

High energy shower (HE) observation : All electrons and high energy shower phenomena of gamma-rays and nuclei are acquired. The high energy shower observation is always activated since this is the trigger mode for the main objectives of CALET. The trigger rate above $10 \mathrm{GeV}$ is $\sim 7.6 \mathrm{~Hz}$ on average [ए]].

Low energy electron (LE) observation: For electron data in the $1 \mathrm{GeV}$ region, this mode can acquire the low energy data efficiently when the geomagnetic cutoff effect is low. It is activated for $90 \mathrm{~s}$ at the highest geomagnetic latitude in the north and south region.

Low energy gamma-ray (LEG) observation: Using a geomagnetic cutoff for charged particles, low energy gamma ray data is acquired efficiently. It is activated in the low geomagnetic latitude region when the geomagnetic latitude is below 20 degree except for the South Atlantic Anomaly (SAA).

Singles run: To check the equipment gain and stability, we collect proton/helium events selectively. It is normally activated during two consecutive orbits ( 3 hours) per day to collect enough statistics to monitor the gain stability.

Ultra-heavy nuclei (UH) observation: A dedicated trigger mode to acquire ultra heavy nuclei penetrating CHD and upper four layers of IMC is implemented. While the main target of the trigger is nuclei over $Z=26$, the trigger threshold is loose enough to trigger on iron. The ultra heavy observation is also always activated because of low trigger rate.

Pedestal run: Pedestal data are periodically acquired (100 events per every 23 minutes ) to avoid the synchronization with the orbital period, 90 minutes.

Aside from the above trigger modes, CGBM is independently operated to protect a detector from high radiation at high geomagnetic latitude and SAA [ㅍ]]. The trigger mode of LEG is automatically activated by the CGBM trigger to detect the gamma-rays over $1 \mathrm{GeV}$.

\section{Data Analysis}

For a calorimeter like CALET, energy calibration is of the utmost importance. The method and associated uncertainties of energy calibration are described and summarized in Ref. [[4]]. Taking into account the detector responses, such as pedestal noise and several calibration uncertainties, detailed calibration achieved a fine energy resolution of $2 \%$ or better in the energy region from $20 \mathrm{GeV}$ to $20 \mathrm{TeV}$ ( $<3 \%$ for $10-20 \mathrm{GeV}$ ). Regarding temporal variations caused during the longterm observation, CALET is calibrated for each detector component by modeling the variations of the MIP peak obtained from non-interacting particles (proton or helium) taken with a dedicated trigger mode. The gain change rate is less than $1 \%$ per month on average for all of the detector components except for a few low-gain channels in CHD, and the variation rate decreases as time advances. The variation rate is less than $0.5 \%$ per month after one year from the start of operations. As another important calibration, the IMC fiber alignment is also calibrated using penetrating particles on orbit, which is very well-consistent with ground calibration results using atmospheric muons. 
The preliminary analysis presented in this paper is based nominally on flight data covering a period of observation from October, 2015 and collected with a dedicated high energy shower trigger. A live time fraction to total time close to $84 \%$ was achieved during this period [ए2]. The stability of the operations on the ISS and the continuous inflight calibrations of the instrument provided an excellent basis for accurate direct measurements of charged and neutral cosmic radiation. In Fig. [1, a distribution of the energy-deposit in TASC is presented to show the coverage of measured energy-region with CALET. It is clearly seen from the smooth distribution that CALET has successfully carried out the measurements from $1 \mathrm{GeV}$ to $1 \mathrm{PeV}$ without any difficulties in the energy scale determination.

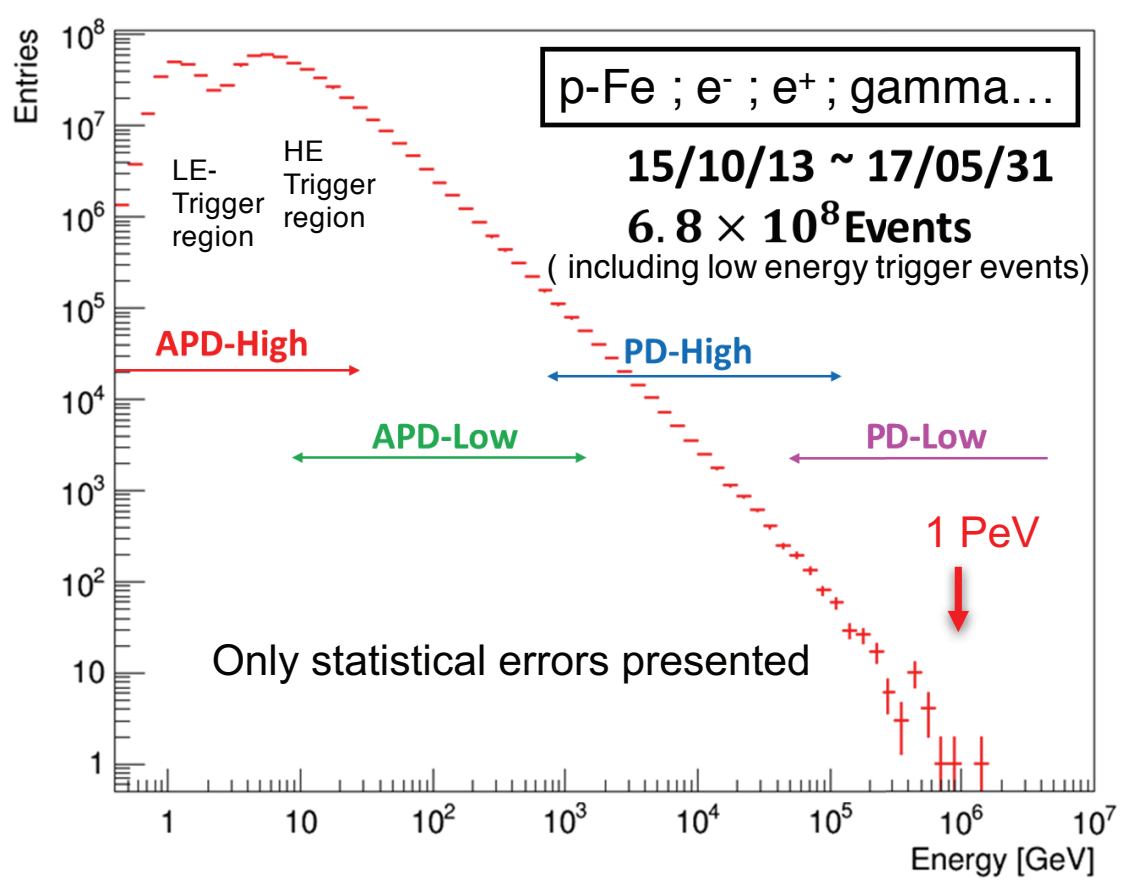

Figure 5: Distribution of the energy-deposit in TASC by observations for 597 days. The horizontal bars indicate the corresponding gain ranges in the signal readout with the Dual PD/APD [ㄷ]].

\subsection{All-Electron Energy Spectrum}

We have analyzed flight data (FD) obtained by the high-energy shower trigger for 536 days to March 31, 2017. The total observational live time is 10,812 hours. The Monte Carlo (MC) program is used to simulate physics processes and detector signals based on the simulation package EPICS [16] (EPICS9.20 / COSMOS8.00), which is updated by the accelerator beam test, with a detailed detector configuration. The MC event samples are generated in order to derive event selection and event reconstruction efficiencies, energy correction factor, and background contamination. Physics analysis is performed as described in the following [[1]].

Track reconstruction: Since some of calibrations and most of the selection parameters depend on the trajectory of an incoming particle, track reconstruction is one of the most important steps in data analysis. As a track reconstruction algorithm, in the present study, we adopt "electromagnetic shower tracking” (EM track) [ए]8], which takes advantage of electromagnetic shower 
characteristics and of the IMC design concept. Thanks to appropriately arranged tungsten plates between IMC layers, shower developments for incoming electrons are clean and stable. By using the well- developed shower core at the bottom IMC layers as the origin of the track finding algorithm, very reliable and highly efficient track reconstruction becomes possible.

Event pre-selection: In order to minimize and accurately subtract proton contamination in electron identification, it is important to apply preselection of well-reconstructed and wellcontained events having unit charge. Furthermore, by removing events that are not included in MC samples, i.e., incidence from zenith angle greater than $90^{\circ}$ and heavier particles, equivalent event samples between FD and MC were obtained in order to be fed into the electron identification. This is the most important purpose of preselection. Preselection consists of offline trigger, the geometrical condition that the reconstructed track must traverse the instrument from CHD top to TASC bottom layer, track quality cut to ensure track reconstruction accuracy, charge selection using CHD, and proper shower-development requirements using likelihood and shower concentration at the IMC bottom layers. Combined efficiency of preselection for electrons is very high: 90 $\%$ above $30 \mathrm{GeV}$ to $3 \mathrm{TeV}, 85 \%$ at $20 \mathrm{GeV}$ and down to $60 \%$ at $10 \mathrm{GeV}$ due to trigger efficiency.

Energy reconstruction: In order to reconstruct the energy of primary electrons, an energy correction function is derived using the electron MC data after preselection. The energy deposit in the detector is obtained as the sum of TASC and IMC, where a simple sum is enough for TASC while compensation for energy deposits in tungsten plates is necessary for IMC. The correction function is then derived by calculating the average ratio of the true energy to the energy deposit sum in the detector. Owing to total absorption, the correction factor is very small, $\sim 5 \%$, up to the $\mathrm{TeV}$ region. Since the energy resolution is sufficient, there is no need to apply energy unfolding.

Electron identification: The last step of event selection is electron identification taking advantage of the shower shape difference between electromagnetic and hadronic showers. We applied two methods, i.e., simple two parameter cuts and multivariate analysis (MVA) to understand systematic effects and stability of the resultant flux. A simple two parameter cut uses $\mathrm{K}$ estimator defined by $\mathrm{K}=\log 10(\mathrm{FE})+\mathrm{RE} / 2 \mathrm{~cm}$, where $\mathrm{RE}$ is the second moment of lateral energy-deposit distribution in the TASC first layer with respect to the shower axis, and FE is the fractional energy deposit of TASC-Y6 layer sum with respect to the TASC total sum. For MVA analysis, we use the multivariate analysis toolkit TMVA [10] to train boosted decision trees (BDT) and to calculate BDT response. The discriminating variables are selected in an energy-dependent manner by using the variables with very good agreement between MC and FD. The discriminating variables above $500 \mathrm{GeV}$ are (1) RE , (2) FE , (3-6) parameters from the fit of longitudinal shower development in TASC, and (7-9) parameters to fit the pre-shower development with an exponential function in IMC. In order to maximize the rejection power against the abundant protons, especially at the highest energy region above $500 \mathrm{GeV}$, MVA has been adopted, while the simple parameter cut was used below $500 \mathrm{GeV}$.

BDT analysis for e/p separation: In the BDT analysis, the whole set of MC data is equally separated into training and test samples. While training is carried out only using the training dataset, test samples are actually used to estimate the efficiency for electrons and contamination of protons. In order to take advantage of the distinctive shape of shower development for electrons, training was carried out separately for each geometry condition and for each finely binned energy region, with a bin-width factor of $10^{1 / 25}$. Examples of BDT response distributions are shown in Fig. 6 , 
which demonstrate the separation power and very good agreements between FD and MC.
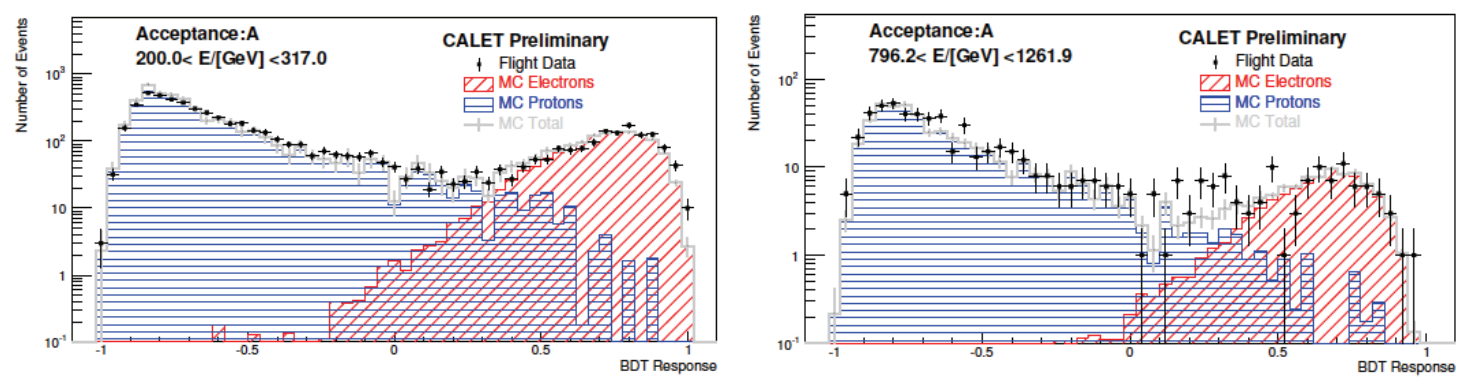

Figure 6: Examples of BDT response distribution in $200<\mathrm{E}<317 \mathrm{GeV}$ (left) and $796<\mathrm{E}<1262 \mathrm{GeV}$ (right).

Subtraction of proton contamination: In order to extract the proton contamination in the final electron sample, template fittings of the K-parameter and BDT response were used, where normalization factors for MC electrons and MC protons are adopted as fitting parameters. The cut position corresponding to $80 \%$ efficiency is determined using the distribution of $\mathrm{MC}$ electrons. The contaminating protons are derived as the expected absolute number of events from the distribution of MC protons and the normalization factor, in order to subtract the background protons, regardless of the spectral shape of the electrons. The resultant contamination ratios of protons in the final electron candidates are 5\% (8\%) in the BDT (K-cut) analysis below $1 \mathrm{TeV}$. The contamination ratio will be much more reduced in further analysis.

Geomagnetic Cutoff Rigidity Measurements: The energy scale calibrated with MIPs is commonly checked in space experiments by analysis of the geomagnetic cut-off energy [20]]. For this study, data samples obtained by the low energy shower trigger $(\mathrm{E}>1 \mathrm{GeV})$ are selected inside an interval of the McIlwain $L$ parameter [ [2]] of 0.95-1.25. By dividing the interval of $L$ into three bins: $0.95-1.00,1.00-1.14$ and 1.14-1.25., different rigidity cut-off regions are selected corresponding to $\sim 15 \mathrm{GV}, \sim 13 \mathrm{GV}$ and $\sim 11 \mathrm{GV}$, respectively. The cut-off rigidity is calculated by using the track trajectry tracing code ATMNC3 [22] and the International Geomagnetic Reference Field, IGRF-12 [23]]. The rigidity cut-off in the electron flux is measured by subtracting correctly the secondary components (re-entrant albedo electrons) while checking the azimuthal distribution in corresponding rigidity regions as shown in the left-hand panel of Fig. $\square$. It is found that the average ratio of the expected to measured cut-off position in the electron flux is $1.035 \pm 0.009$ (stat.), as presented in the right-hand panel of Fig. $\square$. As a result, a correction of the energy scale by $3.5 \%$ was implemented in the analysis.

Flux derivation: The differential flux between energy $E$ and $E+\Delta E[\mathrm{GeV}]$, with bin width $\Delta E[\mathrm{GeV}]$, is given by the following formula:

$$
\Phi(E)=\frac{N(E)-N_{\mathrm{BG}}(E)}{S \Omega \varepsilon(E) T(E) \Delta E(E)},
$$

where $\Phi(E)\left[\mathrm{m}^{-2} \mathrm{sr}^{-1} \mathrm{~s}^{-1} \mathrm{GeV}^{-1}\right]$ is the differential flux, $N(E)$ is the number of electron candidates in the corresponding bin, $N_{\mathrm{BG}}(E)$ is the number of background events estimated as MC protons, $S \Omega$ $\left[\mathrm{m}^{2} \mathrm{sr}\right]$ is the geometrical acceptance, $\varepsilon(E)$ is the detection efficiency for electrons, and $T(E)$ [s] is the observational live time. While $T(E)$ is basically constant, it is necessary to take into account the 

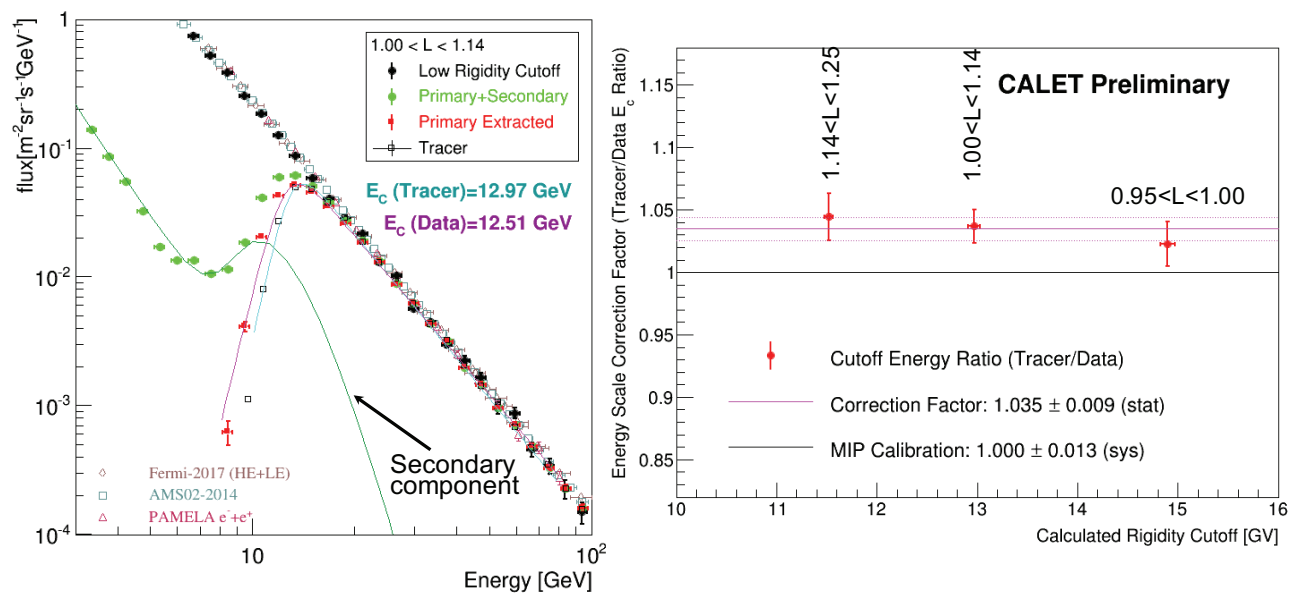

Figure 7: Comparison of measured cutoff rigidity with calculated one (denoted as Tracer) which trace particles in earth's magnetic field (left). Correction factor using the analysis adopted in the left figure for 3 different rigidity cutoff regions (left). The average of correction factors, 1.035, is applied to the energy by MIP calibrations.

cutoff rigidity below $E<30 \mathrm{GeV}$, where the total live time is limited because we use only the data taken with rigidity cutoffs below $6 \mathrm{GV}$. The electron plus positron spectrum measured with CALET is presented in Fig. 8 . Details of the analysis including systematic uncertainties are described in Ref. [ㅁ]]. Regarding the measurements in the $\mathrm{TeV}$ region, note that our constant-efficiency analysis is very important in order to avoid possible efficiency-related systematics.

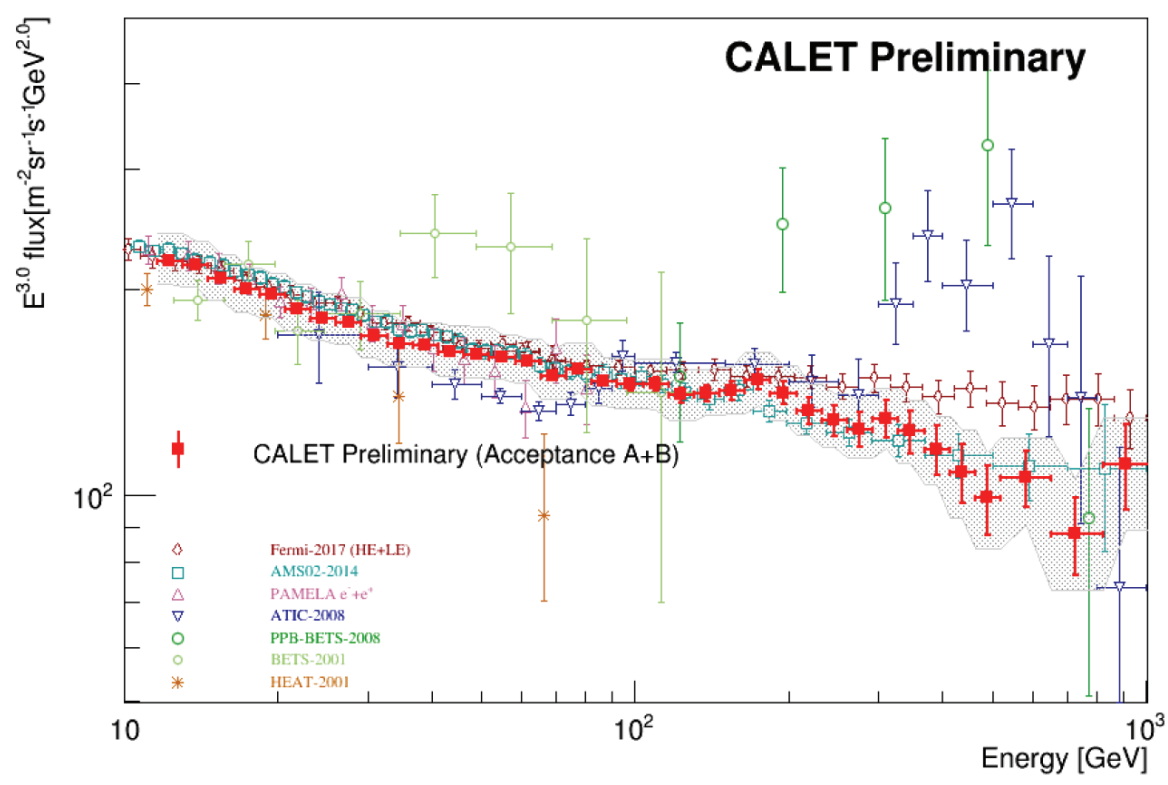

Figure 8: Cosmic-ray all electron spectrum measured by CALET in an energy range from $10 \mathrm{GeV}$ to $1 \mathrm{TeV}$, where systematic errors (not including the uncertainty on the energy scale) are drawn as a gray band. A part of the events, which are fully-contained inside the detector, was used for the analysis, and the number of the events is nearly $50 \%$ of the full data set. Also plotted are measurements in space [24, 25, 26] and from ground based experiments [27, 28]]. 


\subsection{Preliminary Analysis of CR Nuclei}

\subsubsection{Charge Identification and Resolution}

The identification of cosmic nuclei via a measurement of their charge is carried out in CALET with two independent subsystems that are routinely used to cross-calibrate each other: the CHD and the IMC. The latter, being equipped with individually readout scintillating fibers, has a suitable granularity to provide excellent tracking capabilities [29] and to sample the ionization deposits in each layer thereby providing a multiple $\mathrm{dE} / \mathrm{dx}$ measurement with a maximum of 16 samples along the track. The interaction point (IP) is first reconstructed [30] and only the $\mathrm{dE} / \mathrm{dx}$ ionization clusters from the layers upstream of the IP are used. The charge value is evaluated as the truncated mean of the valid samples with a truncation level set at $70 \%$. The non-linear response due to the saturation of scintillation light in the fibers is corrected for by the method described in Ref. [B]].

After application of position and time-dependent calibrations and corrections for each CHD paddle [32] (e.g: gain uniformity, light yield as a function of distance from the PMT, cornerclipping effects, etc.) and of the aforementioned correction for the non-linear response of the scintillator, the information from the two CHD layers is combined into a single charge estimator. Consistency between the charge assignment from two CHD layers is required within $20 \%$. The overall CHD charge resolution (in $\mathrm{Z}$ units) is then plotted in Fig. $\mathbf{Q}$ (a) showing a linear increase as a function of the atomic number from $\leq 0.1$ for protons to 0.3 for iron. For the IMC, although the photo-statistics available at single fiber level are about one order of magnitude lower than a single CHD layer, the charge measurement, thanks to the multiple sampling, can achieve an adequate performance as shown in Fig. $Q(b)$ where the IMC charge resolution is plotted as a function of the atomic number $\mathrm{Z}$ after restoration of a linear response as explained above. An adequate separation power is provided for instance for boron and carbon with a charge resolution of 0.14 in the CHD and 0.2 in the IMC.

By correlating the two charge measurements, as in the example shown in Fig. Q $($ (d) where the IMC charge is plotted versus the CHD charge (hereafter referred to as Z-plane), well separated charge peaks emerge on top of a low and flat background for light nuclei candidates from boron to neon. This is also true for the lightest elements proton and helium as shown in Fig. Q(c). Under these conditions a clean charge separation is possible allowing for a robust rejection power of $\mathrm{He}$ candidates in the selected proton sample as explained in the following.

\subsubsection{Preliminary Analysis of Proton and Light Nuclei}

Due to the limited equivalent thickness of $1.3 \lambda_{I}$ of the IMC+TASC calorimeter module, only $80 \%$ of protons interact on average in CALET. The HE trigger efficiency rises very slowly from a threshold close to $7 \mathrm{GeV}$ and reaches $10 \%$ at $50 \mathrm{GeV}$ which is the lowest energy point we have included in the present analysis. Neither geomagnetic nor solar modulation effects play a significant role above this energy.

Charge selection of proton events is performed by application of an elliptical cut in the Zplane. This cut has been studied to allow for a mild residual energy dependence of the position of the CHD proton charge peak most probably related to the energy increase of backscattering from the TASC. A residual fractional contamination of helium falls below the percent level above 200 $\mathrm{GeV}$ is shown. 


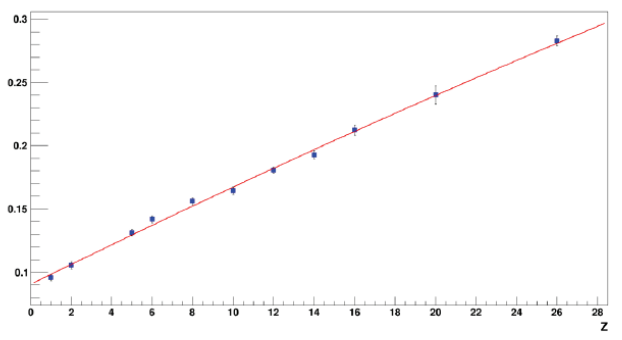

(a)

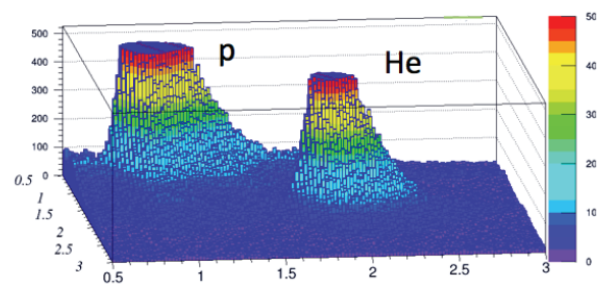

(c)

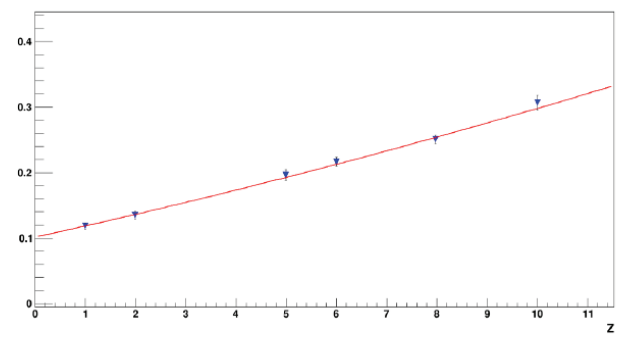

(b)

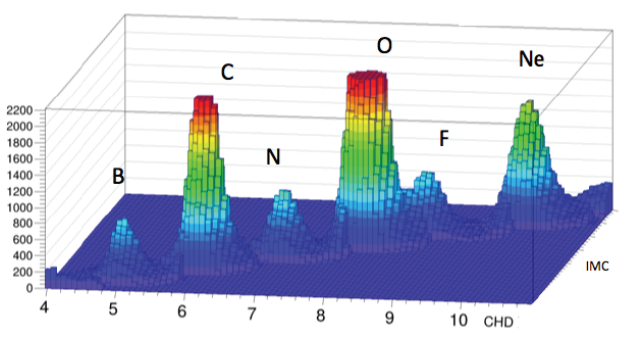

(d)

Figure 9: (a) Charge resolution (in units of the elementary charge) vs. atomic number $Z$ for the two CHD layers combined; (b) IMC charge resolution for a selection of light elements; (c) Charge distribution of proton and helium candidates in the Z-plane (see text); (d) Charge distributions for B, C, N, O, F, Ne candidates in the Z-plane. The charge peaks of the most abundant elements have been chopped off in the picture to improve the visibility of the less abundant ones.

Rejection of electrons is at present achieved by using a cut on the fraction of energy deposited in the bottom layer of the TASC with respect to the total energy. The proton efficiency for this cut is $90 \%$ with a mild dependence on energy. Most of the electron background is below $50 \mathrm{GeV}$, therefore there is no need to introduce a more severe selection at the expense of proton efficiency. Above $50 \mathrm{GeV}$ the remaining contamination of electrons in the proton sample is close to the percent level.

In contrast to electrons, the measured energy of protons is significantly lower than the primary particle's energy (typically $\sim 40 \%$ ) and smeared by the calorimeter resolution for hadronic showers causing a migration of events from a given energy bin into the nearby ones. A standard technique of energy unfolding to recover the true value of the primary particle's energy is to construct, using MC data, an energy overlap matrix.

The main efficiency corrections (trigger, tracking, charge selection, electron-ID) are individually studied as a function of the MC true energy using three different MC simulation packages: FLUKA, EPICS and GEANT4. Tracking efficiency reaches a $\geq 95 \%$ plateau at $1 \mathrm{TeV}$ while it has a value of $90 \%$ at $100 \mathrm{GeV}$ with room for improvement at lower energies. Charge selection efficiency is almost flat at $95 \%$ above $50 \mathrm{GeV}$ slowly decreasing to $90 \%$ at $10 \mathrm{TeV}$. The electron rejection efficiency is $93 \%$ at $100 \mathrm{GeV}$ and $90 \%$ at $10 \mathrm{TeV}$. The HE trigger efficiency (normalized to all events in the acceptance including non interacting protons) is $40 \%$ at $1 \mathrm{TeV}$. The probability of a wrong assignment of the acceptance type, as provided by the reconstruction of the track and of the shower axis, is taken into account in the analysis. 
The preliminary CALET proton spectrum determined as discussed above is presented in Fig.5(a) in Ref. [B]]. Application of the standard techniques to investigate the stability of the measurement by scanning the parameter space of various systematic uncertainty is underway. The assessment of the systematic uncertainties in the proton analysis (not presented here) will improve in time along with the steady increase of the available statistics and more refined understanding of the instrument.

\subsubsection{Light Nuclei}

The analysis of the absolute and relative fluxes of light elements is underway. It requires, for each chemical species under study, the subtraction of the residual background contamination in the selected sample. The main sources of background include: (i) charge changing interactions in the apparatus (mainly by nuclear spallation); (ii) wrong charge assignment due to the presence of backscattered radiation from the calorimeter. The former typically produces a migration of event candidates toward lower atomic numbers while the latter works in the opposite direction because of the extra energy deposits collected along the track on top of the ionization generated by the primary particle. The measurement of the relative elemental abundances requires a correction for the above effects. This is usually encoded into an array that contains the expected transition probabilities among the different nuclei as derived from the MC simulations. Also, energy deconvolution has to be carried out. At the present preliminary stage of the analysis we only show the $\mathrm{dN} / \mathrm{dE}$ distributions of light nuclei candidates after a simple data pre-selection requiring a high energy trigger (HET) and a well reconstructed track inside Acceptance-type A. Charge selection is then performed in the IMC vs CHD charge plane by applying a circular cut of radius 0.8 (in $\mathrm{Z}$ units) around the nominal charge $Z$ for each element with $2<Z<11$. The selected candidates from proton to oxygen are plotted in Fig.5(b) in Ref. [B] ] as a function of the reconstructed calorimetric energy (in GeV/A units).

\subsubsection{Heavy Nuclei}

On-board trigger for heavy nuclei (heavy ions) are detected by the High Energy shower trigger (HE trigger), over $10 \mathrm{GeV}$. While penetrating light nuclei like protons and helium are not triggered in this mode, heavy ions with $\mathrm{Z}>8$ that interact in deep layers are detected because the $\mathrm{dE} / \mathrm{dx}$, which is proportional to $Z^{2}$, is large enough to exceed the trigger threshold. The trigger efficiency for ions with $Z>8$ is therefore almost $100 \%$.

To maintain good charge resolution and remove events that interact in the CHD, we require consistency in the CHD and IMC layers before estimating the charge of each event. Events where the difference of CHD-X and CHD-Y is less than $15 \%$ are selected. Consistency of the charge average using the 1st and 2nd (x,y) IMC layers to the average obtained using 3rd and 4th (x,y) IMC layers is also required. Efficiency of these cuts is $65-70 \%$ for heavy nuclei $(Z>8)$ with little energy dependence. Charge determinations in CALET are primarily based on the signals from the CHD paddles on the reconstructed tracks.

We applied an unfolding procedure for the derivation of the primary energy spectrum, based on Bayesian method as described in Ref. [33]. Response functions were made from detailed Monte Carlo simulations. Selection efficiencies of the charge consistency cuts and charge 
identification method were taken into account in the unfolding process. Contaminants from the neighboring charged nuclei were also de-convolved.

The initial power law index in the unfolding procedure was set to -2.60 . In order to study the uncertainty in the unfolding procedure due to the initial spectral index used, two other indices were tested: 2.50 and -2.70 . In this index range, the uncertainty is less than $2 \%$, which is significantly smaller than the statistical errors at high energies.

Figure 10 shows the preliminary energy spectra of neon, magnesium, silicon and iron up to $100 \mathrm{TeV}$ derived from CALET observations. The spectra were plotted above $20 \mathrm{GV}$ in order to avoid the effects of geomagnetic rigidity cutoffs.

\subsubsection{Ultra Heavy Nuclei}

By a preliminary CALET UHCR analysis for the first 18 months of CALET data [34] , the Z-even relative abundance $\left({ }_{26} \mathrm{Fe}=1\right)$ is obtained as shown in Fig. $\square$ for the events that passed CALET UH trigger, had reconstructed trajectories, were within $60^{\circ}$ of vertical, passed the CHD charge consistency cuts, and which had a geomagnetic vertical cutoff rigidity $4 \mathrm{GV}$. Using the vertical rigidity cut on such a wide incidence-angle acceptance leaves some spillover from the more abundant lower-Z peaks that obscure ${ }_{30} \mathrm{Zn}$ and ${ }_{31} \mathrm{Ga}$ as shoulders, but one sees peaks for ${ }_{32} \mathrm{Ge},{ }_{34} \mathrm{Se},{ }_{36} \mathrm{Kr}$ and ${ }_{38} \mathrm{Sr}$.

\subsection{Gamma-ray Observations}

CAL operates on-orbit in two trigger modes, the HE trigger and the LEG trigger. The HE trigger is operated with a nearly $100 \%$ duty fraction, being disabled only for passage through the SAA, and enables measurement of electrons and gamma-ray events with energies above 10 $\mathrm{GeV}$. The LEG trigger is sensitive to primary energies down to $1 \mathrm{GeV}$, but is only active at low geomagnetic latitudes (where the rigidity cutoff is used to reduce contamination from charged particles) and following gamma-ray bursts (GRB) seen by CGBM. 
We apply a gamma-ray selection by tracking pair creation events in IMC for the flight data. The gamma-ray event selection used in this analysis is described in Ref. [36]. According to the simulation study that has generated events around the instrument isotropically, we estimate that the highest gamma-ray efficiency is achieved around $10 \mathrm{GeV}$ with an efficiency of $50 \%$ relative to a geometrical factor of $420 \mathrm{~cm}^{2} \mathrm{sr}$, which is the $100 \%$ efficiency case, by applying the event selections described above. The effective areas for various incident angles are calculated as a function of energy. Analysis presented here is optimized to enlarge the field-of-view as wide as possible, so we used a geometrical cut which is optimized for point source analysis. Then we also have to reject gamma-ray candidates which come through the ISS structures (such as solar panels) to remove events generated by cosmic-ray interactions with these structures, which produce event clusters clearly visible in our field-of-view [37]. Incident gamma-ray energies were estimated based on the deposited energy in CAL considering the geometry conditions.

Our long-term CAL observation of Galactic diffuse gamma-rays in the low-energy gamma-ray mode clearly indicates the Galactic plane enhancement and some strong sources on the count map as shown in Fig. 12 .

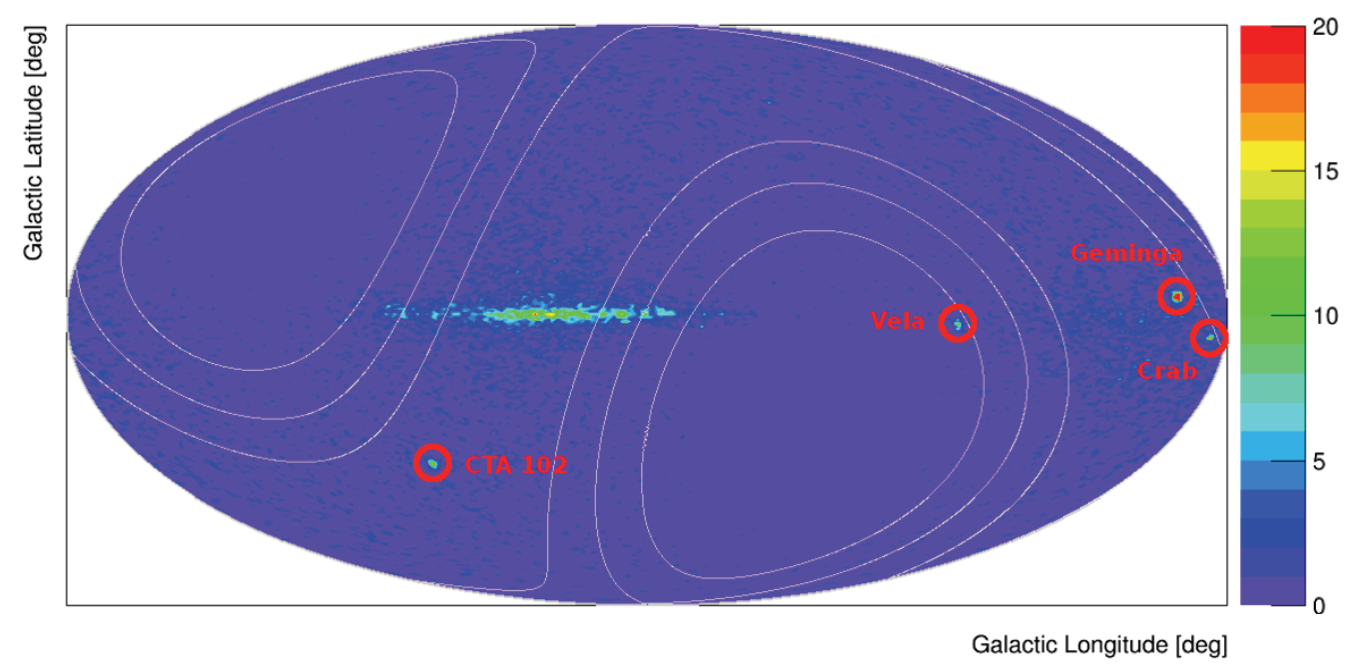

Figure 12: Count map of gamma-ray candidates obtained in the low-energy gamma-ray mode during the time period from 2015 October 13 to 2017 May 31 expressed in the Galactic coordinates. The color bar is shown in unit of counts per $2.56 \times 10^{-4} \mathrm{sr}\left(\sim 0.52^{\circ}\right.$ radius circle $)$. Also shown by contours are the exposure calculated for this period $(25,50$ and $75 \%$ of the full level). Some strong sources are also observed.

\subsubsection{Point sources}

The Crab can be clearly seen in Fig. 12 and can be used to confirm the in-flight angular resolution performance. For a selection of events from the region of the Crab, the angular distances of the events from the known source position as given by SIMBAD distribute with a measured $\sigma_{P S F}=0.4^{\circ}$. The fluxes are measured for the Crab and Geminga. Photons associated with the sources are chosen to lie within a distance of $5 \sigma_{P S F}$ of the known source positions. The differential flux is calculated by summing the photon candidates weighted by the CAL detection efficiency and scaling to the exposure on the sky for the source position and energy bin width. For the Crab, the 
exposure is calculated to be $\sim 4 \times 10^{8} \mathrm{~cm}^{2} \mathrm{~s}$, with 46 photon candidates associated with the source position. For Geminga, the exposure is $\sim 5 \times 10^{8} \mathrm{~cm}^{2} \mathrm{~s}$, with 119 associated photon candidates. The flux is consistent with the Fermi-LAT 3FGL spectra [38] in both Geminga and Crab.

Transient activity from the bright radio blazar CTA 102 [BG] was also observed by CAL. The flux was measured in the CAL as the AGN begins to rise in 2016/11, reaches a peak in 2016/12, and begins to fade in 2017/01. Smaller flares are seen in 2017/02 and 2017/04. Analysis is ongoing to increase the field of view of CAL for CTA 102 and similar transients.

\subsubsection{Galactic diffuse component}

Galactic latitude distribution of gamma-rays is obtained by summing over the Galactic longitude range, $-80^{\circ}<l<80^{\circ}$, by observation in the time period from 2015 October 13 to 2017 May 31. By comparison with the Galactic diffuse radiation model developed by the Fermi-LAT team, we found general consistency between the CALET observation and the model.

\section{Summary and Future Prospects}

Preliminary analysis based on a sub-sample of the data collected by the observation since October, 2015 on the ISS has shown the capability of CALET to extend substantially the energy reach of the direct measurement of charged cosmic rays. The steady observations of CALET gives us another opportunity to observe the GW EM counterpart with the CALET Gamma-ray Burst Monitor ( not described in this paper, see Ref. [143] ) and Calorimeter as reported in Ref. [40, [36]. Moreover, we have been contributing to Space Weather Monitoring by detecting the relativistic electron precipitation events associated with solar flares [4]]. The so far excellent performance of CALET and the outstanding quality of the data suggest that a 5-year observation period might provide a wealth of new interesting data to resolve the mysteries of the high-energy universe.

\section{Acknowledgements}

We gratefully acknowledge JAXA's contributions to the development of CALET and to the operations onboard the ISS. We also wish to express our sincere gratitude to ASI and NASA for their support of the CALET project. This work was supported in part by a JSPS Grant-in-Aid for Scientific Research (S) (no. 26220708) and by the MEXT-Supported Program for the Strategic Research Foundation at Private Universities (2011-2015) (No. S1101021) at Waseda University.

\section{References}

[1] J. Nishimura, et al., Astrophys. J. 238, 394 (1980).

[2] T.Kobayashi, Y.Komori, K.Yoshida, and J.Nishimura, Astrophys. J. 601, 340 (2004).

[3] O.Adriani, et al., Nature 458, 607 (2009).

[4] L. Accardo, et al., Phys. Rev. Lett. 113, 121101 (2014).

[5] P.S. Marrocchesi, et al., Nucl. Instrum. Meth. A, 659 (2011) 477.

[6] P.S. Marrocchesi for the CALET collaboration, Proceedings of Science (ICRC2013) 362 (2013).

[7] T. Niita, S. Torii, Y. Akaike, Y. Asaoka, K. Kasahara, et al., Advances in Space Research, 55 (2015) 2500 .

[8] Y. Akaike for the CALET collaboration, Proceedings of the 32nd ICRC (Beijing) Vol.6 346 (2011). 
[9] L. Pacini and Y. Akaike for the CALET collaboration, Proceedings of Science (ICRC2017) 163 (2017).

[10] M.Mori for the CALET collaboration, Proceedings of Science (ICRC2013) 248 (2013).

[11] Y.Asaoka for the CALET collaboration, Proceedings of Science (ICRC2015) 603 (2015).

[12] S.Ozawa and Y.Asaoka for the CALET collaboration, Proceedings of Science (ICRC2017) 165 (2017).

[13] K. Yamaoka for the CALET collaboration, Proceedings of Science (ICRC2017) 613 (2017).

[14] Y. Asaoka, Y. Akaike, Y. Komiya, R. Miyata, S.Torii, et al., Astroparticle Physics, 91,1 (2017).

[15] R. Miyata, Y. Asaoka, S. Torii for the CALET collaboration, Proceedings of Science (ICRC2017) 207 (2017).

[16] K.Kasahara, Proceedings of 24th ICRC, Vol.1 (1995) 399. http://cosmos.n.kanagawa-u.ac.jp/EPICSHome/

[17] Y.Asaoka for the CALET collaboration, Proceedings of Science (ICRC2017) 205 (2017).

[18] Y.Akaike, et al., Proceedings of Science (ICRC2013) 726 (2013).

[19] A. Hocker Proc. Sci. , ACAT2007 040.

[20] M. Ackermann, et al., Atroparticle Physics 35, 346 (2012).

[21] D.F.Smart and M.A.Shea, Advances in Space Research 36, 2012 (2005).

[22] M.Honda, T.Kajita, K.Kasahara and S.Midorikawa, Physical Review D 70, 043008 (2004).

[23] E. Thébault, et al., Earth, Planets and Space 67:79 (2015).

[24] M. Aguilar, et al., Phys. Rev. Lett. 113, 221102 (2014).

[25] S. Abdollahi, et al., Phys. Rev. D 95082007 (2017).

[26] O. Adriani, et al., La Rivista del Nuovo Cimeto, to be pubilshed. (private communication)

[27] F.Aharonian, et al., Phys. Rev. Lett. 101, 261104 (2008).

[28] F.Aharonian, et al., Astron Astrophysics. 308, 561 (2009).

[29] P. Maestro and N.Mori for the CALET collaboration, Proceedings of Science (ICRC2017) 208 (2017).

[30] P. Brogi for the CALET collaboration, Proceedings of Science (ICRC2017) 595 (2017).

[31] P.S. Marrocchesi for the CALET collaboration, Proceedings of Science (ICRC2017) 156 (2017).

[32] Y.Komiya, S.Torii, G.Bigonigiari and Y.Asaoka, for the CALET collaboration, Proceedings of Science (ICRC2017) 206 (2017).

[33] Y.Akaike for the CALET collaboration, Proceedings of Science (ICRC2017) 181 (2017).

[34] B.Rauch and Y.Akaike for the CALET collaboration, Proceedings of Science (ICRC2017) 180 (2017).

[35] R. P. Murphy, et al., Astrophys. J. 831 (2016) 2083.

[36] M.Mori and Y.Asaoka for the CALET collaboration, Proceedings of Science (ICRC2017) 637 (2017).

[37] N.Cannady for the CALET collaboration, Proceedings of Science (ICRC2017) 720 (2017).

[38] F. Acero, et al., Astrophys. J. Supplement Series, 218:23 (2015).

[39] S.Ciprini, et al., ATel No.9869 (2016).

[40] O. Adriani, et al., (CALET collaboration), Astrophysical Journal Letters, 829, L20 (2016).

[41] R. Kataoka, et al., Geophysical Research Letters, 43, 4119 (2016). 